Matute, Jose A., Mauricio Marcano, Asier Zubizarreta, and Joshue Perez. "Longitudinal Model Predictive Control with Comfortable Speed Planner." 2018 IEEE International Co nference on Autonomous Robot Systems and Competitions (ICARSC) (April 2018) 60 - 64. doi:10.1109/icarsc.2018.8374161.

https://doi.org/10.1109/icarsc.2018.8374161 


\title{
Longitudinal Model Predictive Control with Comfortable Speed Planner
}

\author{
Jose A. Matute ${ }^{\mathrm{a}, \mathrm{b}}$, Mauricio Marcano ${ }^{\mathrm{a}, \mathrm{b}}$, Asier Zubizarreta ${ }^{\mathrm{b}}$, Joshue Perez ${ }^{\mathrm{a}}$ \\ ${ }^{a}$ Industry and Transport Division, Tecnalia Research \& Innovation, San Sebastian, Spain \\ ${ }^{b}$ Automatic Control and System Engineering Department, University of the Basque Country, Bilbao, Spain \\ joseangel.matute@tecnalia.com, mauricio.marcano@tecnalia.com, asier.zubizarreta@ehu.eus, joshue.perez@tecnalia.com
}

\begin{abstract}
Guaranteeing simplicity and safety is a real challenge of Advanced Driver Assistance Systems (ADAS), being these aspects necessary for the development of decision and control stages in highly automated vehicles. Considering that a human-centered design is generally pursued, exploring comfort boundaries in passenger vehicles has a significant importance. This work aims to implement a simple Model Predictive Control (MPC) for longitudinal maneuvers, considering a bare speed planner based on the curvature of a predefined geometrical path. The speed profiles are constrained with a maximum value at any time, in such way that total accelerations are lower than specified constraint limits. A double proportional with curvature bias control was employed as a simple algorithm for lateral maneuvers. The tests were performed within a realistic simulation environment with a virtual vehicle model based on a multi-body formulation. The results of this investigation permits to determine the capabilities of simplified control algorithms in real scenarios, and comprehend how to improve them to be more efficient.

Index Terms-Model Predictive Control, Simulation Environment, Automated Driving.
\end{abstract}

\section{INTRODUCTION}

In the path of automated driving an efficient mobility is a clear purpose of the intelligent transportation industry, optimizing the use of vehicles and infraestructure to provide safety and comfort within the driving experience. It has been determined that passenger perception of comfort is very subjective and vary due several factors, being the users driving style pointed as the most important aspect [1]. The magnitude of comfort is inversely propotional to vehicle's acceleration and its relative change (jerk), hence the higher these values are, the more discomfortable should be the driving [2].

One of the typical requirements in vehicles automation is the capability to follow specified routes being time effective, comfortable and considering limits for traveling velocities. The implementation of a speed planner that consider smoothness in acceleration and jerk transition in curve paths is a very useful way to improve comfort. Some strategies have been employed as Jerk Limitation Method [3] or Quintic Bezier Curve Method [4]. However these procedures mean additional efforts that could be avoided using alternative strategies in longitudinal control algorithms.

Model Predictive Control (MPC) is a concept based on optimal control theory, where future behaviors of a test platform can be predicted from its model. A reduced design

Copyright notice: 978-1-5386-5346-6/18/\$31.00 2018 IEEE model with this technique permits to obtain acceptable results, having the versatility to specify constraints as acceleration and jerk responses [5]. The present work aims to use a MPC for longitudinal maneuvers, in order to take advance of its prediction capacity without complex speed planner calculation.

This paper is organized as follows: Section II present the control algorithm used for longitudinal control and its relationship with the speed planner proposed. Section III describes the simulation environment where tests are developed considering the virtual vehicle used as test platform. Section IV shows the obtained results followed by a proper discussion. Finally the Section V summarizes the most important ideas.

\section{CONTROL Algorithm}

The design of control algorithms for path tracking applications based on passenger comfort is a very useful technique on intelligent transportation systems. In this section, a novel approach based on a speed planner, with special attetion in standarization [2], and the proposed longitudinal MPC control approach is presented.

Both algorithms are developed under a general automated framework [6], a six block architecture which includes the following modules: acquisition, perception, communication, decision, control and actuation. This framework has been previously used for different automated vehicle applications such as path tracking lateral [7], validation of longitudinal controllers [8] and overtaking maneuvers [9]. This architecture is suitable for implementing a path tracking controller module taking into account the passenger comfort.

\section{A. Speed Planner}

The speed planner is based in offline and online modules as shown in Figure 1. Offline module comprises the local map and static speed planner. The local map stage used in this work is part of a decision module, where Bezier curves have been used to generate a soft path along a route including straight lines, turns and a lane change (Figure 2) [10]. Additionally, the local map stage calculates the curvature $(k)$ of each segment of the road as parametric curves [11] delivering this information along with $n$ local map points $(x, y)$ to static speed planner.

The objective of the proposed approach is to assure passenger comfort during autonomous driving. The comfort parameter is obtained through a combination of longitudinal $\left(a_{x}\right)$, 


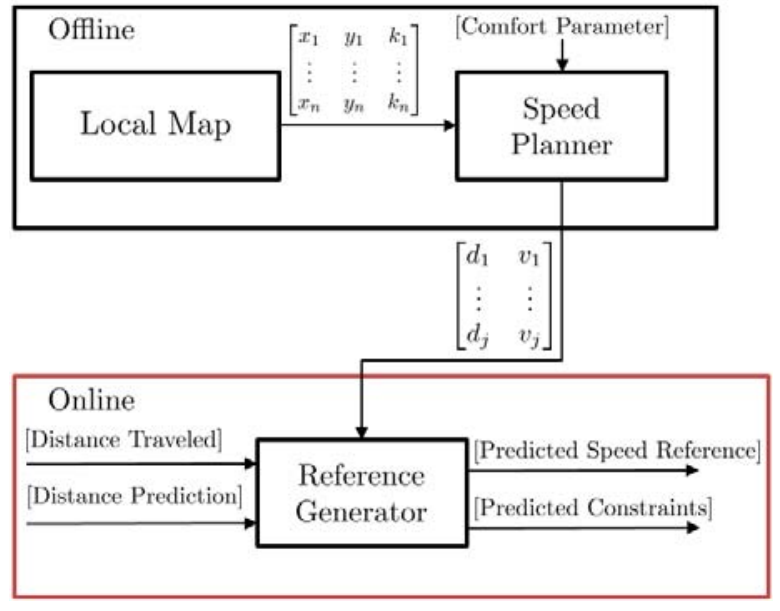

Fig. 1. Speed Planner Framework

lateral $\left(a_{y}\right)$ and vertical $\left(a_{z}\right)$ accelerations, considering certain weights $\left(n=n_{x}=n_{y}=1.4\right)$ [2]:

$$
a_{w}=\sqrt{n_{x} \cdot a_{x}^{2}+n_{y} \cdot a_{y}^{2}+a_{z}^{2}}
$$

Numerical values have been assigned to $a_{w}$ indicating several levels of umcomfortability: $0.315 \mathrm{~m} / \mathrm{s}^{2}$ (Not), $0.63 \mathrm{~m} / \mathrm{s}^{2}$ (a little), $1.0 \mathrm{~m} / \mathrm{s}^{2}$ (fairly), $1.6 \mathrm{~m} / \mathrm{s}^{2}$ (uncomfortable) and $2.5 \mathrm{~m} / \mathrm{s}^{2}$ (very). The comfort parameter and maximum speed are related using the curvature of each road segment defined as $k$ :

$$
V_{\max }=\sqrt{\frac{a_{w}}{n \cdot k}}
$$

According to Equation 2, the speed reference is calculated based on the curvature of the road limiting the speed to 15 $\mathrm{km} / \mathrm{h}$. The speed profile is generated as a function of the distance traveled $d_{t}$ from a starting point $\left(x_{0}, y_{0}\right)$. Figure 2 shows the curvature profile of the test scenario. From that information a speed profile is generated using the Equation 2.

Once the speed profile is obtained (as a 2D vector relating distance traveled $(d)$ against speed $(v)$ ), it is sent to the online reference generator, which supply the MPC with the longitudinal speed references (see Section 2.B). While the vehicle is moving it calculates the value of $d_{t}$ by integrating the current speed, this value is passed to the reference generator along with the prediction of the longitudinal displacement calculated by the controller. This allows to calculate the appropriate speed reference according to the vehicle position and its future predictions based on distance traveled instead of time.

\section{B. Model Predictive Control}

Model Predictive Control approaches are based on the use of a mathematical representation of the model to predict the future behavior of a system in a finite time horizon. The control action is obtained by minimizing a cost function which can include constraints. The approach depends on a precise characterization of the system in order to work correctly.
However, real-time implementation is possible, updating the current state and solving the problem each sample time, thus allowing the use of an approximated model. With this in mind, a brief explanation of the control problem is presented.

A real-time implementation allows to work with a tripleintegrator model based on the speed $\left(v_{l}\right)$, acceleration $\left(a_{l}\right)$ and jerk $\left(j_{l}\right)$ of the vehicle seen as a particle in longitudinal displacement $\left(d_{l}\right)$. The representation of the system in the classical state-space description is shown in Equation 3.

$$
\left[\begin{array}{l}
\dot{d}_{l} \\
\dot{v}_{l} \\
\dot{a}_{l}
\end{array}\right]=\left[\begin{array}{lll}
0 & 1 & 0 \\
0 & 0 & 1 \\
0 & 0 & 0
\end{array}\right]\left[\begin{array}{l}
d_{l} \\
v_{l} \\
a_{l}
\end{array}\right]+\left[\begin{array}{l}
0 \\
0 \\
1
\end{array}\right] j_{l}
$$

In this model the jerk acts as the control variable, however interest is over the predicted states of the longitudinal acceleration which will be the input to the vehicle. Following classical state space MPC formulation [12], the control law can be calculated from the minimization of the following cost function.

$$
J=\sum_{s=1}^{N_{h}}\left\|v_{l}(t+s \mid t)-v_{r e f}(t+s \mid t)\right\|^{2}
$$

Where $t$ is the current time, $s$ is the sample index starting from time $t, N_{h}$ is the number of samples of the prediction, $v_{l}(t+s \mid t)$ represents $s^{t h}$ longitudinal speed prediction calculated at time $t$, while $v_{r e f}$ is the reference speed including its prediction delivered by the speed planner. Finally the vector solution $\overline{\boldsymbol{a}}_{l}$ is found and the command selector choose to accelerate or decelerate depending on the sign; it also choose the prediction index to use as control action to the simulated vehicle. Future speed references of the online module of the speed planner are calculated based on the past prediction of the MPC as it can be shown in Figure 3 where the controller general framework and speed profile generator are detailed.

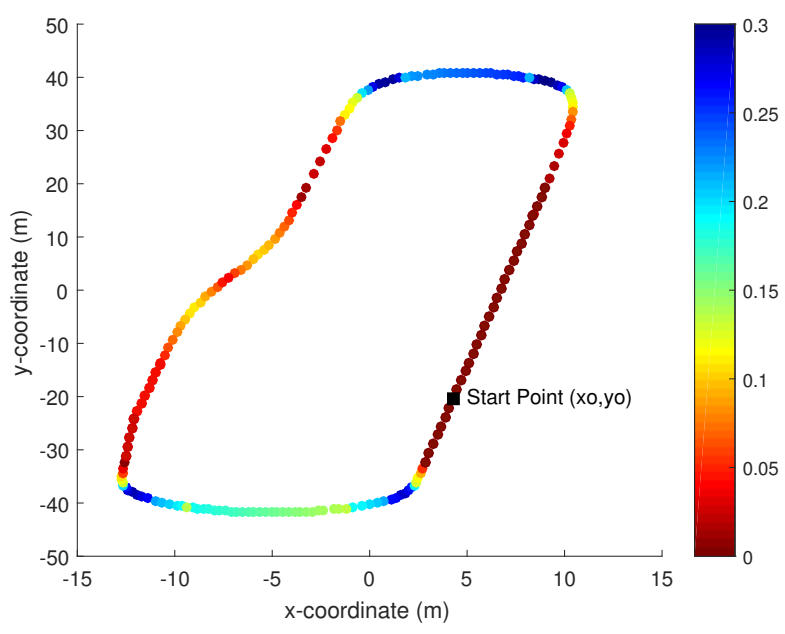

Fig. 2. Curvatures in predefined circuit 


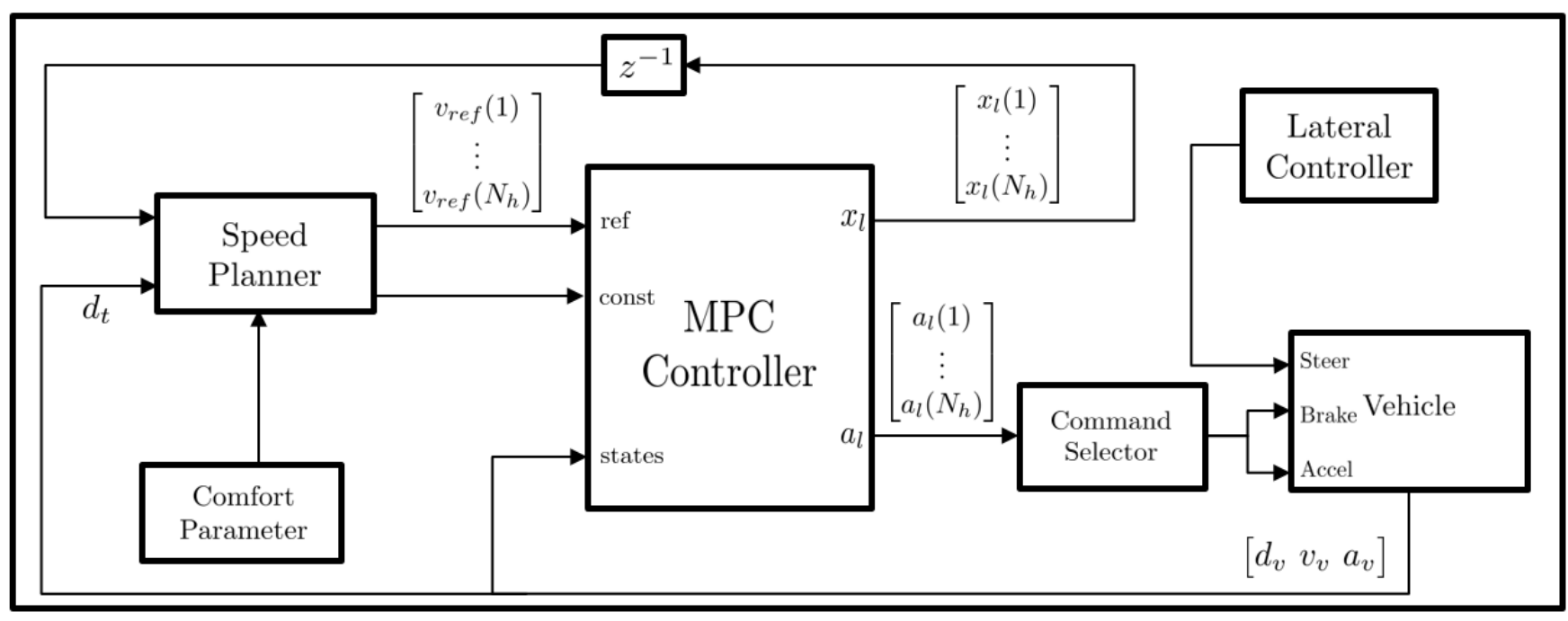

Fig. 3. Model Predictive Control Framework integrated with Speed Planner

Lastly, constraints values of the controllers are fixed for acceleration and jerk, however the speed constraints are based on the speed planner predictions. $L$ represents an error allowed for the solution of the speed vector.

$$
\begin{aligned}
v_{r e f}(t+s)-L & \leq v_{l}(t+s \mid t) \leq v_{r e f}(t+s)+L \\
a_{\min } & \leq a_{l}(t+s \mid t) \leq a_{\max } \\
j_{\min } & \leq j_{l}(t+s \mid t) \leq j_{\max }
\end{aligned}
$$

For solving previous constrained optimization problem, the MPC solver ACADO toolkit [13] is used. It is a self-contained library based on $\mathrm{C}++$ designed to solve linear and non-linear models under multi-objective optimization functions.

\section{SIMULATION ENVIRONMENT}

The developed approach is tested in Dynacar simulation environment [14]. Dynacar features a vehicle physical model based on multi-body formulation with a framework integrated in Matlab-Simulink. The motion of the chassis and wheels are considered into a model of fourteen degrees of freedom, being possible for the chassis to translate and rotate in any cartesian axis, and for each wheel to move in vertical direction and rotate around its own axis. The suspension system degrees of freedom are considered as macro-joints and their behavior is modelled through look-up tables. The position and orientation of wheels are important to calculate the suspension response, therefore the values within the tables strongly depend on the steering input and the roll angle [15].

The vehicle physical model has been designed to provide each wheel with steering angle, and traction or braking torque, being this an versatile to users due the possibility to test control algorithms fitted to a model within a realistic environment. The physical behavior of tire-ground contact was obtained through the Magic Formula Tire Model [16].

In the following subsections the powertrain and brake models implemented will be briefly detailed. The parameters
TABLE I

VirTuAl Vehicle PARAMETERS

\begin{tabular}{ll}
\hline Mass $(\mathrm{kg})$ & 611.500 \\
CG location $\boldsymbol{x}, \boldsymbol{y}, \boldsymbol{z}(\mathbf{m})$ & $-0.928,0.000,0.488$ \\
Wheelbase $(\mathbf{m})$ & 1.686 \\
Trackwidth $(\mathbf{m})$ & 1.094 \\
Inertia $\boldsymbol{I} \boldsymbol{x}, \boldsymbol{I} \boldsymbol{y}, \boldsymbol{I} \boldsymbol{z}\left(\mathbf{k g}-\mathbf{m}^{2}\right)$ & $243.175,430.166,430.166$ \\
Front wheel radius $(\mathbf{m})$ & 0.265 \\
Rear wheel radius $(\mathbf{m})$ & 0.281 \\
\hline
\end{tabular}

of the virtual vehicle used as simulation testbed in this work are depicted in Table I.

\section{A. Powertrain model}

Fig. 7 shows the simplified powertrain model employed. A synchronous electric motor, a gearbox and a mechanical differential are the only elements involved. The drivetrain type is rear-wheel-drive of locked axle. The model behavior is linearly dependent of the throttle pedal position $\left(u_{t}\right)$ which goes from 0 to 1 .

The selected electric motor can develop $57 \mathrm{~N}-\mathrm{m}$ of constant traction torque $\left(T_{e}\right)$ before surpasing a speed of $220 \mathrm{rad} / \mathrm{sec}$, providing a decreasing torque as the motor speed $\left(\omega_{e}\right)$ increases up to $785 \mathrm{rad} / \mathrm{sec}$. A regenerative braking torque $\left(T_{b}\right)$ is considered into the model, which delivers $8 \mathrm{~N}$-m constantly

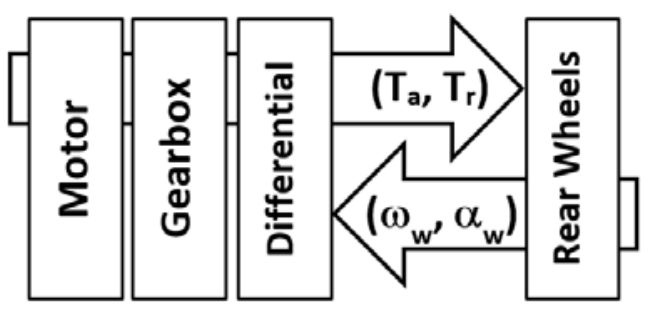

Fig. 4. Powertrain model 
if the motor speed is higher than $50 \mathrm{rad} / \mathrm{sec}$ and $u_{t}$ is zero. This estimation was obtained from movement tests at several speeds. Both traction and regenerative braking characteristics are typical in this kind of motor [17], [18].

The automatic gearbox has a reduction of $1: 9.23$ permiting reverse, neutral and drive positions. The differential changes the power flow direction in 90 degrees to the rear wheels with a 1:1 transmission ratio. Compact equations for traction $\left(T_{a}\right)$ and regenerative breaking $\left(T_{r}\right)$ torques are shown in Eqs. 6-7.

$$
\begin{gathered}
T_{a}=T_{e} \eta_{g d} N_{g d} \operatorname{sign}\left(u_{t}\right)-\left(\left(I_{e}+I_{g}\right) N_{g d}^{2}+I_{d} N_{d}^{2}\right) \alpha_{w} \\
T_{r}=T_{b} \eta_{g d} N_{g d}
\end{gathered}
$$

Where $\eta_{g d}$ is the combined efficiency of the driveline, $N_{d}$, $N_{d}$ and $N_{g d}$ are the gearbox, differential and combined transmission ratio of the driveline, and $I_{e}, I_{g}$ and $I_{d}$ are the inertias of engine, gearbox and differential. The angular acceleration of the rear wheels $\left(\alpha_{w}\right)$ is necessary to calculates the loss of tractive force due to the inertia of driveline components [19].

\section{B. Brake model}

The braking behavior is related with brake pedal position $\left(u_{b}\right)$ that goes from 0 to 1 . The model consists of a reduced single-circuit [20] where the braking torque depends of the pressure over the braking disc exerted by the wheel cylinder $\left(P_{w}\right)$ as shown in Fig. 5. As the dynamic of a typical brake line considers a lag associated to the hydraulic system $\left(\tau_{b}\right)$, the pressure can be modeled as a discrete transfer function with a defined time span $\left(t_{s}\right)$ as depicted in Eq. 8 .

$$
P_{w}(z)=\left(K_{c} t_{s} u_{b}\right) /\left(\tau_{b} z+\left(t_{s}-\tau_{b}\right)\right)
$$

Control valves in brake systems are generally used to adjust pressure in rear brakes and prevents tires lock. This behavior is introduced keeping a brake gain $\left(K_{b} i\right)$ constant for rear wheels when its value exceeds a selected limit. An hyperbolic tangent function is used to control the orientation of the braking torque, being this calculation dependent of the wheel speed $\left(\omega_{w}\right)$ as can be seen in Eq. 9

$$
T_{b}=P_{w} K_{b} \tanh \left(\omega_{w}\right)
$$

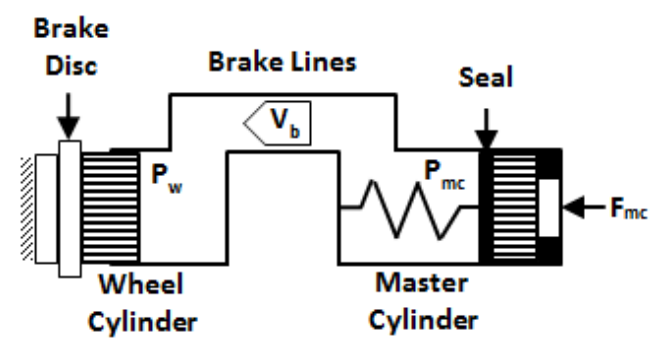

Fig. 5. Brake model
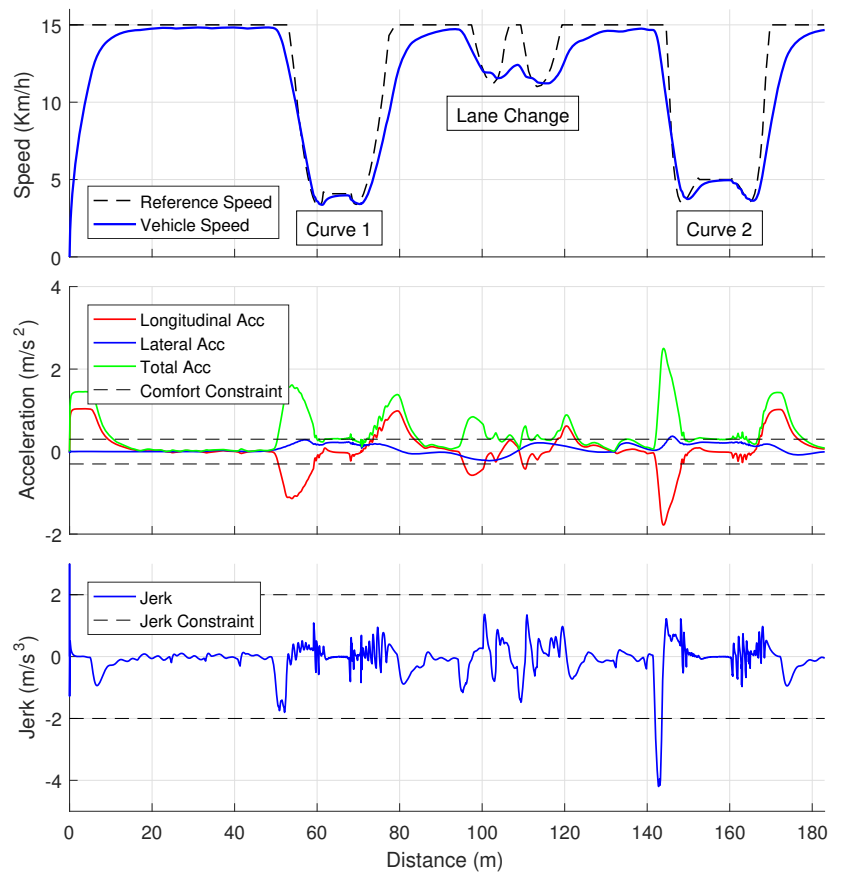

Fig. 6. Not uncomfortable Accelerations $\left(0.3 \mathrm{~m} / \mathrm{s}^{2}\right)$

\section{TEST AND RESUlts}

In this section the proposed approach will be tested in several scenarios, considering the test circuit depicted in Figure 3. This circuit is suitable to evaluate the performance of the proposed approach in different urban conditions, such as straight lines, turns with different radios and lane change.

For the developed tests, the speed profiles are calculated in such way that total accelerations are lower than specified constraint limits, going from not uncomfortable $\left(0.315 \mathrm{~m} / \mathrm{s}^{2}\right)$ to very uncomfortable $\left(2.5 \mathrm{~m} / \mathrm{s}^{2}\right)$ level. A permissible speed error band of $0.1 \mathrm{~km} / \mathrm{h}$ around the speed profile depicted as $L$ in Equation 5, accelerations limits of $-4 \mathrm{~m} / s^{2}$ and $1 \mathrm{~m} / \mathrm{s}^{2}$, and jerk boundaries of $-2 \mathrm{~m} / s^{3}$ and $2 \mathrm{~m} / s^{3}$ were considered as constraints within the MPC. The optimization problem was solved with a sample time of $1 \mathrm{~s}$ and 10 total samples.

Two tests were performed in the environment detailed in Section II for evaluating the performance of the MPC speed tracker with a speed planner. Test responses for comfort parameters levels of Not Uncomfortable $\left(a_{w}=0.3 \mathrm{~m} / \mathrm{s}^{2}\right)$ and Very Uncomfortable $\left(a_{w}=2.5 \mathrm{~m} / s^{2}\right)$ are detailed as follows.

In both cases the speed of the vehicle follows the reference. The MPC anticipates future changes at the entrance and exit of turns and lane change situation. For Very Uncomfortable level the lane change does not appears due higher accelerations are permitted. Some inconsistencies are observed during curve paths (close to $d=50 \mathrm{~m}$ and $d=140 \mathrm{~m}$ ), and is because the vehicle faces turns at high velocity and violent transitions are needed to follow the speed reference. 


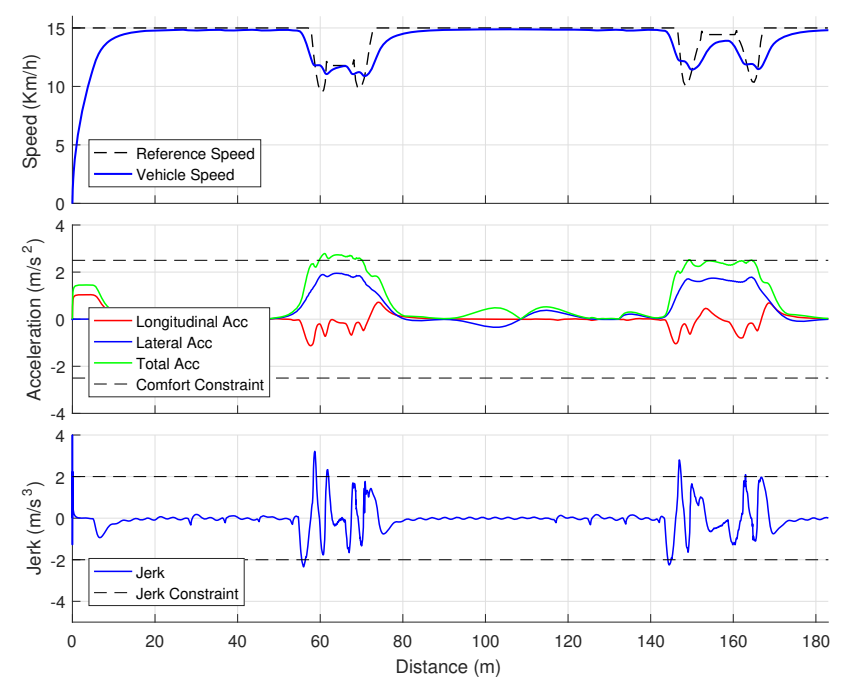

Fig. 7. Very Uncomfortable Accelerations $\left(2.5 \mathrm{~m} / \mathrm{s}^{2}\right)$

The acceleration component which comprises longitudinal and lateral values succeeded while the vehicle circulates through curve paths. For Not Uncomfortable level, some peaks appear in longitudinal accelerations in order to follow the speed planner before entrance and after exit of curves, having this an impact in the level of comfort in total acceleration value. However, the study is focused to achieve a desirable comfort level exclusively during turns to adapt the safety user perception under this circumstance. Peaks of acceleration are not observed in Very Uncomfortable level because speed does not need a violent transitions from curves to straight paths.

Jerk values are generally within limits in both comfort level cases. The solver surpasses jerk constraint in order to achieve reference speed under very short periods of time, where greater priority is given to the MPC optimization function.

\section{COnClusions}

The results of this investigation permits to determine the capabilities of simplified control algorithms in real scenarios, and comprehend how to improve them to be more efficient. An MPC speed tracker was evaluated and showed a good performance while working together with an speed planner based on curvature and comfort of passengers. At different comfort levels the controller behaves properly and total acceleration in curves were kept close to the reference limit. In future investigations this simplified MPC will be evaluated and configurated on real test platforms.

\section{ACKNOWLEDGMENT}

Authors want to acknowledge their organization. This project has received funding from the Electronic Component Systems for European Leadership Joint Undertaking under grant agreement No 737469 (AutoDrive Project). This Joint Undertaking receives support from the European Unions Horizon 2020 research and innovation programme and Germany, Austria,
Spain, Italy, Latvia, Belgium, Netherlands, Sweden, Finland, Lithuania, Czech Republic, Romania, Norway. This work was developed at Tecnalia Research \& Innovation facilities supporting this research.

\section{REFERENCES}

[1] J. Karjanto and N. Yusof, "Comfort determination in autonomous driving style," in AutomotiveUI'15, September 2015.

[2] "Mechanical vibration and shock - Evaluation of human exposure to whole-body vibration - Part 1: General requirements," standard, International Organization for Standardization, Geneva, CH, May 1997.

[3] J. Villagra, V. Milans, J. Prez, and J. Godoy, "Smooth path and speed planning for an automated public transport vehicle," Robotics and Autonomous Systems, vol. 60, no. 2, pp. 252 - 265, 2012.

[4] D. Gonzlez, V. Milans, J. Prez, and F. Nashashibi, "Speed profile generation based on quintic bezier curves for enhanced passenger comfort," in 2016 IEEE 19th International Conference on Intelligent Transportation Systems (ITSC), pp. 814-819, Nov 2016.

[5] F. Borrelli, P. Falcone, T. Keviczky, J. Asgari, and D. Hrovat, "Mpcbased approach to active steering for autonomous vehicle systems," International Journal of Vehicle Autonomous Systems, vol. 3, 2005.

[6] D. González and J. Pérez, "Control architecture for cybernetic transportation systems in urban environments," in Intelligent Vehicles Symposium (IV), 2013 IEEE, pp. 1119-1124, IEEE, 2013.

[7] R. Lattarulo, J. Pérez, and M. Dendaluce, "A complete framework for developing and testing automated driving controllers," IFACPapersOnLine, vol. 50, no. 1, pp. 258-263, 2017.

[8] M. Marcano, J. A. Matute, R. Lattarulo, E. Mart1, and J. Pérez, "Low speed longitudinal control algorithms for automated vehicles in simulation and real platforms,"

[9] R. Lattarulo, M. Marcano, and J. Pérez, "Overtaking maneuver for automated driving using virtual environments," in International Conference on Computer Aided Systems Theory, pp. 446-453, Springer, 2017.

[10] D. González, J. Pérez, R. Lattarulo, V. Milanés, and F. Nashashibi, "Continuous curvature planning with obstacle avoidance capabilities in urban scenarios," in Intelligent Transportation Systems (ITSC), 2014 IEEE 17th International Conference on, pp. 1430-1435, IEEE, 2014.

[11] D. G. Bautista, Functional architecture for automated vehicles trajectory planning in complex environments. $\mathrm{PhD}$ thesis, PSL Research University, 2017.

[12] E. F. Camacho and C. B. Alba, Model predictive control. Springer Science \& Business Media, 2013

[13] B. Houska, H. J. Ferreau, and M. Diehl, "Acado toolkitan open-source framework for automatic control and dynamic optimization," Optimal Control Applications and Methods, vol. 32, no. 3, pp. 298-312, 2011.

[14] I. Iglesias-Aguinaga, A. Martin-Sandi, and A. Pea-Rodriguez, "Vehicle modelling for real time systems application. the virtual rolling chassis," DYNA, vol. 88, no. 2, pp. 206-215, 2013.

[15] J. Cuadrado, D. Vilela, I. Iglesias, A. Martin, and A. Pea, "A multibody model to assess the effect of automotive motor in-wheel configuration on vehicle stability and comfort," ECCOMAS Thematic Conference Multibody Dynamics 2013, pp. 457-458, 2013.

[16] H. B. Pacejka, "Preface," in Tyre and Vehicle Dynamics (Second Edition) (H. B. Pacejka, ed.), pp. v - vii, Oxford: Butterworth-Heinemann, second edition ed., 2006.

[17] R. N. Jazar, Vehicle Dynamics. Theory and Application. Business Media New York 2014, Springer Science, 2014.

[18] J. Nadeau, P. Micheau, and M. Boisvert, "Ideal regenerative braking torque in collaboration with hydraulic brake system," in 2017 Twelfth International Conference on Ecological Vehicles and Renewable Energies (EVER), pp. 1-5, April 2017.

[19] T. Gillespie, Fundamentals of Vehicle Dynamics. Premiere Series Bks, Society of Automotive Engineers, 1992.

[20] J. C. Gerdes and J. K. Hedrick, "Brake system modeling for simulation and control," Journal of Dynamic Systems, Measurement, and Control, vol. 121 , no. 3, pp. 496 - 503, 1999. 